Cahiers
Recherche
surles Droits Fondamentaux

\section{Cahiers de la recherche sur les droits} fondamentaux

17 | 2019

La motivation des actes administratifs. Le droit français à la lumière du droit administratif comparé

\title{
La motivation des actes administratifs individuels en droit grec
}

Vassiliki Kapsali

\section{(2) OpenEdition}

\section{Journals}

Édition électronique

URL : https://journals.openedition.org/crdf/3735

DOI : $10.4000 /$ crdf.3735

ISSN : 2264-1246

Éditeur

Presses universitaires de Caen

Édition imprimée

Date de publication : 31 décembre 2019

Pagination : 87-98

ISBN : 978-2-84133-960-0

ISSN : 1634-8842

Référence électronique

Vassiliki Kapsali, «La motivation des actes administratifs individuels en droit grec », Cahiers de la recherche sur les droits fondamentaux [En ligne], 17 | 2019, mis en ligne le 06 février 2021, consulté le 14 novembre 2022. URL : http://journals.openedition.org/crdf/3735 ; DOI : https://doi.org/10.4000/crdf. 3735 


\title{
La motivation des actes administratifs individuels en droit grec
}

\author{
Vassiliki KAPSALI \\ Professeure assistante de droit public à la faculté de droit de l'université Neapolis Pafos (Chypre)
}

I. La perception de la motivation des actes individuels sous un prisme essentiellement contentieux
A. La théorie jurisprudentielle des actes nécessitant une motivation en raison de leur nature
B. La nature particulière de l'exigence de motivation des actes individuels

II. La place secondaire réservée à la motivation formelle des actes individuels
A. Le désintérêt pour l'aspect non contentieux de la motivation
B. L'apport limité de l'article 17 du Code de procédure administrative non contentieuse

Outre le quarantième anniversaire de la loi française $\mathrm{n}^{\circ} 79-587$ du 11 juillet 1979 relative à la motivation des actes administratifs et à l'amélioration des relations entre l'administration et le public ${ }^{1}$, l'année 2019 marque également les vingt ans du Code grec de procédure administrative non contentieuse ${ }^{2}$. Selon son exposé des motifs ${ }^{3}$, le Code grec aspirait, assez classiquement pour un texte de ce type, à clarifier les relations qui se nouent entre l'administration et le public lors de l'élaboration des actes administratifs, de façon à répondre aux impératifs d'un État de droit contemporain, tels que le renforcement de la qualité, de la transparence et de l'efficacité des services administratifs ainsi que la garantie du respect de l'égalité et des droits individuels. Ledit Code s'inscrivait donc globalement dans le même esprit que celui qui a animé l'évolution du droit français en matière de procédure administrative non contentieuse à partir de la fin des années 1970 et jusqu'à sa codification récente par l'ordonnance $\mathrm{n}^{\circ}$ 2015-1341 du 23 octobre 2015 relative aux dispositions législatives du Code des relations entre le public et l'administration.

S'agissant du traitement de la question de la motivation des actes administratifs, les droits français et grec ont eu pour point de départ commun le principe de non-motivation. En France, avant 1979, le secret administratif était la règle 4 et l'exigence de motivation formelle connaissait "peu de faveur » dans la procédure administrative non contentieuse ${ }^{5}$. Le Conseil d'État affirmait le principe selon lequel il n'y a pas de motivation sans texte ${ }^{6}$, tandis que des exceptions au principe de non-motivation des actes individuels résultaient des textes de manière

1. Désormais codifiée dans le Code français des relations entre le public et l'administration.

2. Introduit en vertu de l'article 1 de la loi 2690/1999 ( $\Phi E K A^{\prime}, \mathrm{n}^{\circ} 45,9$ mars 1999 [ФEK $A^{\prime}$ est le journal officiel de la République hellénique dans lequel sont publiées (notamment) les lois]).

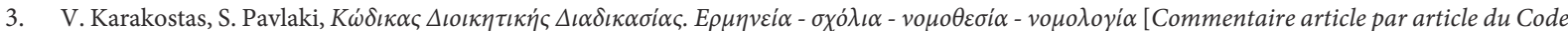
de procédure administrative non contentieuse], Athènes, Nomiki Vivliothiki, 2009, p. 1-2.

4. D'autant plus que l'accès aux documents administratifs était lui aussi limité avant la loi nº 78-753 du 17 juillet 1978.

5. G. Isaac, La procédure administrative non contentieuse, Issy-les-Moulineaux, LGDJ, 1968 (issu d'une thèse de doctorat en droit, université de Toulouse), p. 540 .

6. À titre indicatif, voir CE, 30 avril 188o, Harouel et Morin c. ministre de la Guerre; CE, Ass., 16 décembre 1955, Dame Bourobka; CE, 24 avril 1964, Delahaye. 
sporadique et désordonnée ${ }^{7}$ En Grèce, avant 1999, le Conseil d'État grec (CEgr) reconnaissait également le principe de non-motivation des actes administratifs individuels $^{8}$. La non-motivation a historiquement constitué un élément distinctif des actes administratifs par rapport aux décisions de justice, dont la motivation est obligatoire depuis la Constitution monarchique de $1844^{9}$. Des dispositions textuelles imposaient, exceptionnellement, l'indication formelle des motifs pour certaines catégories d'actes individuels, comme les sanctions disciplinaires et d'autres actes défavorables pour les fonctionnaires, les refus de communication des documents administratifs ou les restrictions à la propriété ${ }^{10}$.

En vertu respectivement des lois de 1979 et de 1999 susmentionnées, la France a introduit une inflexion importante du principe de non-motivation des actes individuels, alors que la Grèce a opté, tout du moins à première vue, pour le renversement de celui-ci, puisque le Code grec de procédure administrative non contentieuse, dans son article 17-1, énonce généreusement que «l'acte administratif individuel doit comporter une motivation, consistant dans la constatation de la réunion des conditions légales pour son édiction ${ }^{11}$. Cette disposition, qui semble instituer une obligation générale de motivation des actes individuels en comblant ainsi une lacune dans la protection procédurale des individus vis-à-vis de l'administration, n'est en réalité pas simple à saisir. Pour la comprendre et pour en mesurer la portée, il faut retracer l'histoire jurisprudentielle de l'exigence de motivation et expliquer la conception assez particulière de la notion même de motivation retenue en droit grec et cristallisée d'ores et déjà dans le Code de procédure administrative non contentieuse.

En effet, avant l'intervention de la loi de 1979 en France et du Code de 1999 en Grèce, les juges administratifs des deux pays avaient développé certaines techniques contentieuses pour assurer le contrôle efficace des motifs des actes administratifs, que le principe général de non-motivation rendait difficile. Avec la jurisprudence Barel et ses suites ${ }^{12}$, le juge français du recours pour excès de pouvoir s'était octroyé le pouvoir inquisitorial de connaître les motifs des actes individuels en cours d'instance contentieuse, en réclamant à l'administration la communication de tous les documents susceptibles d'établir sa conviction et de permettre la vérification des allégations du requérant concernant la régularité des motifs de l'acte litigieux. Qui plus est, le juge administratif français n’hésitait pas à contrôler, s'agissant des actes discrétionnaires, la qualification juridique des faits, l'exactitude matérielle des faits ainsi que l'appréciation administrative de ces derniers, par le biais notamment de la technique de l'erreur manifeste d'appréciation ${ }^{13}$. Le Conseil d'État grec, dans le cadre du recours pour excès de pouvoir, avait pour sa part élargi les contours de son pouvoir de contrôle sur l'activité administrative, et surtout sur les motifs des actes discrétionnaires, par le biais de la théorie jurisprudentielle dite des actes nécessitant une motivation en raison de leur

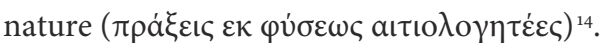

Ces techniques ont été instaurées dans l'objectif de faciliter l'exercice du contrôle juridictionnel sur la légalité interne des actes individuels et, par extension, dans le but de garantir la sauvegarde des droits et intérêts particuliers face au danger de l'arbitraire administratif. Si les droits français et grec ne reconnaissaient pas la motivation expresse des actes individuels en tant qu'obligation générale, les juges n'ont toutefois jamais renoncé au contrôle des motifs de ces actes. En France au moins, la distinction entre la motivation formelle et les motifs des actes administratifs était déjà nette. Comme le soulignait M. Letourneur dans ses conclusions sur l'arrêt Barel,

La règle suivant laquelle les actes administratifs n'ont pas à être motivés a pour conséquence de supprimer à leur égard le vice de forme tiré du défaut de motifs; elle n'a ni pour but ni pour effet de soustraire l'administration à l'obligation de faire connaître au juge [...] les motifs des actes attaqués devant lui ${ }^{15}$.

En somme, sous le règne du principe de nonmotivation des actes individuels, les droits français et grec avaient élaboré deux outils principaux en vue d'assurer aux administrés une protection juridique adéquate: l'un consistait dans l'introduction occasionnelle d'une exigence formelle de motivation de l'acte administratif, l'autre dans le développement de techniques contentieuses facilitant l'accès du juge aux motifs dans le cadre du recours pour excès de pouvoir. Or le parallélisme entre les deux ordres juridiques en matière de motivation des actes individuels s'arrête ici, car les progrès ultérieurs réalisés par les droits administratifs français et grec ont été inégaux du point de vue du degré dans lequel l'exigence d'extériorisation des motifs administratifs a pu être détachée de son utilité et vocation contentieuses et du degré dans lequel les administrés sont devenus les bénéficiaires de la garantie

7. J. Manesse, Le problème de la motivation des décisions administratives, thèse de doctorat en droit, université Paris 2, 1976, p. 58 sq.

8. À titre indicatif, voir CEgr, 2429/1964, 845/1971 et 1460/1975.

9. Voir actuellement l'article 93-3 de la Constitution de 1975.

10. Pour plus d'informations, voir A. Tsoutsos, «La motivation des actes administratifs », Revue internationale des droits de l'homme, 1970, p. 126 sq.;

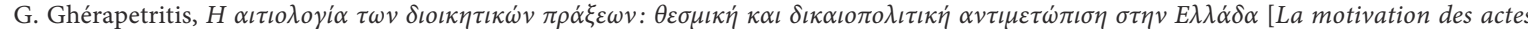
administratifs en Grèce: approche institutionnelle et juridico-politique], Athènes - Komotini, Sakkoulas, 1998, p. 73 sq.

11. Toutes les traductions du grec sont les nôtres.

12. CE, Ass., 28 mai 1954, Barel; CE, 21 décembre 1960, Vicat-Blanc; CE, 26 janvier 1968, Maison Genestal.

13. Sur ce sujet, voir M. Letourneur, "L'étendue du contrôle du juge de l'excès de pouvoir», Études et documents du Conseil d'État, $\mathrm{n}^{\circ}{ }^{18}$, 1962 , p. 51 sq.; B. Pacteau, Le juge de l'excès de pouvoir et les motifs de l'acte administratif, Clermont, Université de droit et de science politique, 1977 (issu d'une thèse de doctorat en droit, université Bordeaux 1).

14. Pour davantage de détails, voir infra I.A.

15. CE, Ass., 28 mai 1954, Barel, concl. M. Letourneur, Revue du droit public, 1954, p. 521. 
de la motivation aussi en leur qualité de destinataires des actes individuels et non seulement en tant que justiciables.

Plus précisément, en France, la mise en place au profit des administrés d'un droit à la motivation formelle est apparue opportune en dépit de la confiance affirmée du système français dans la garantie juridictionnelle des droits et libertés ${ }^{16}$. La communication des motifs au juge et la motivation formelle ne sont ni équivalentes ni interchangeables, mais elles fonctionnent sur des registres distincts et concourent à des objectifs différents. Selon les termes de G. Isaac,

La motivation est et doit rester une règle essentielle de la Procédure Administrative Non Contentieuse; si elle facilite, de surcroît, le contrôle du juge, il ne faut pas prendre cet effet secondaire pour le contenu même de la motivation ${ }^{17}$.

De plus,

[...] l'obligation de motiver ne constitue pas seulement une garantie de forme, mais bien une garantie de fond; elle a, en effet, une influence sur le contenu de l'acte lui-même, en interdisant dans une large mesure la précipitation et l'erreur ${ }^{18}$.

C'est donc en prenant pleine conscience du rôle protecteur pour les administrés, dès la phase administrative décisionnelle, de la motivation formelle que le droit français, en vertu de la loi du 11 juillet 1979, a prévu pour la motivation des actes individuels un essor indépendant du seul domaine contentieux ${ }^{19}$.

En droit grec, au contraire, si la théorie jurisprudentielle des actes nécessitant une motivation en raison de leur nature a contribué à renforcer la protection juridictionnelle des droits et libertés des administrés vis-à-vis du pouvoir administratif, elle a été en même temps la cause principale de l'occultation de l'intérêt de l'extériorisation non contentieuse des motifs administratifs. En effet, la motivation formelle a été la victime du succès de cet outil contentieux, un succès qui a favorisé l'idée d'une prévalence de la protection juridictionnelle des droits des administrés par rapport aux garanties non contentieuses préalables. Partant, le droit grec n'a jamais abandonné le prisme essentiellement contentieux à travers lequel il perçoit la motivation des actes individuels (I) et il a accordé une moindre importance à la motivation formelle en tant que garantie de la procédure administrative non contentieuse (II).

\section{La perception de la motivation des actes individuels sous un prisme essentiellement contentieux}

Le Conseil d'État grec, qui a commencé son activité en 1929, a entendu pallier les obstacles que créait pour le contrôle juridictionnel le principe de non-motivation en élaborant la théorie des actes nécessitant une motivation en raison de leur nature, lesquels devaient se juxtaposer à ceux soumis à la motivation en vertu de dispositions textuelles (A). Conçue comme un outil permettant l'extension du contrôle juridictionnel sur les motifs de l'activité de l'administration, la motivation requise en raison de la nature de l'acte revêt une nature juridique particulière (B).

\section{A. La théorie jurisprudentielle des actes nécessitant une motivation en raison de leur nature}

Le principe de non-motivation des actes individuels obstruait surtout le contrôle juridictionnel des actes issus de l'exercice d'un pouvoir discrétionnaire, pris au sens d'une appréciation plus ou moins étendue portée par l'administration sur les faits de l'affaire. Dans le cadre du recours pour excès de pouvoir, les actes discrétionnaires étaient en principe susceptibles d'un contrôle restreint, qui se limitait à la vérification du détournement de pouvoir

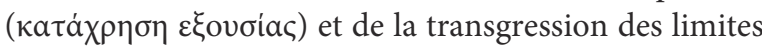

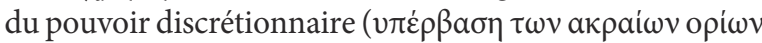

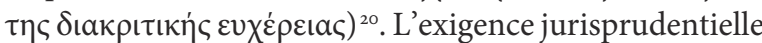
de motivation dégagée de la nature de l'acte déféré au contrôle juridictionnel a donc principalement visé les actes discrétionnaires, l'arrêt 59/1930 du Conseil d'État grec étant considéré comme l'arrêt de principe en la matière ${ }^{21}$. Selon ses termes,

La motivation formelle des actes administratifs est nécessaire si elle est prescrite par la loi. Lorsque l'absence de motivation empêche l'exercice du contrôle sur un acte discrétionnaire de l'administration et que le caractère justifié de l'acte ne se dégage ni de l'activité administrative dans son ensemble ni des documents fournis, alors même que cet acte est, de par sa nature, susceptible de motivation, [...] l'acte en cause doit être motivé afin de permettre la vérification de la bonne application de la loi.

16. En ce sens, voir G. Isaac, La procédure..., p. 545 sq.; R. Hostiou, Procédure et formes de l'acte administratif unilatéral en droit français, Issy-lesMoulineaux, LGDJ, 1975, p. 172 sq. ; et J. Manesse, Le problème de la motivation..., p. 318 sq.

17. G. Isaac, La procédure..., p. 554 .

18. Ibid., p. 547. Sur les diverses fonctions de la motivation formelle, voir aussi C. Wiener, «La motivation des décisions administratives en droit comparé», Revue internationale de droit comparé, 1969, p. 780 sq.

19. La commission du rapport et des études du Conseil d'État, dans son Rapport sur la motivation expresse obligatoire de nouvelles catégories d'actes administratifs, 1978 (texte dactylographié), p. 3, relevait également que «la motivation expresse des actes administratifs se combine avec la garantie que constituent les recours contentieux, mais elle en est indépendante».

20. CEgr, Ass., 620/1933 : «Les actes discrétionnaires sont susceptibles de contrôle et d'annulation en cas de détournement de pouvoir mais aussi lorsqu'ils ont été pris à la suite d'un pouvoir discrétionnaire exercé de manière non conforme à la conviction d'un homme sage [...] et contraire à la bonne administration".

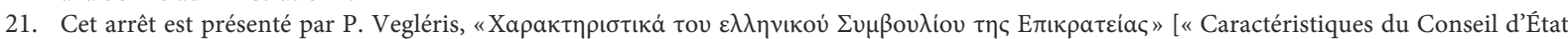

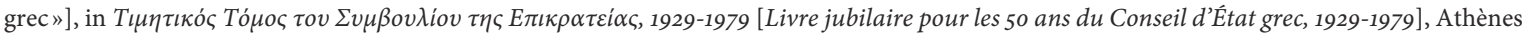
- Komotini, Sakkoulas, 1979-1982, p. 29 sq. 
Depuis, le Conseil d'État grec n'a cessé d'affirmer l'existence d'une exigence de motivation à l'égard de très nombreux actes individuels discrétionnaires se fondant sur leur nature, de sorte que la jurisprudence en cause s'est développée en une théorie jurisprudentielle fondamentale du contentieux administratif grec. Le contrôle de

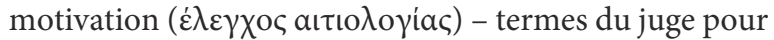
désigner le contrôle qu'il exerce sur les motifs des actes concernés - a servi de contrepoids au pouvoir croissant de l'administration et de garantie protectrice des administrés, dans leurs droits et libertés, face au pouvoir administratif. La catégorie des actes nécessitant une motivation en raison de leur nature a fini par acquérir une étendue considérable.

En effet, l'existence d'une telle exigence de motivation a pu être affirmée à propos de très nombreux actes individuels relevant de domaines variés de l'activité administrative ${ }^{22}$. Les actes concernés sont surtout les actes discrétionnaires et, parmi ceux-ci, principalement les actes défavorables et les actes dérogatoires ${ }^{23}$. La catégorie des actes discrétionnaires couvre, entre autres, des actes de tutelle et d'exercice du pouvoir hiérarchique, des actes pris par des organismes collégiaux, consultatifs ou non, et des actes d'évaluation des candidats pour l'accès aux emplois publics ou pour la promotion des fonctionnaires. Le Conseil d'État grec a considéré comme étant des actes défavorables pour les administrés en particulier des sanctions, disciplinaires ou non, des retraits d'actes favorables, des actes rejetant des recours administratifs, des actes imposant des restrictions aux libertés publiques ainsi que des rejets de demandes. Il a pu qualifier de dérogatoires des actes par lesquels est modifiée une pratique administrative constante, des actes par lesquels l'autorité compétente prend une décision contraire à un avis préalable, des actes en désaccord avec les éléments du dossier ou encore des actes émis en application d'une disposition dérogatoire.

La qualification d'un acte individuel comme nécessitant une motivation en raison de sa nature est opérée in concreto et de façon casuistique ${ }^{24}$. Le Conseil d'État grec n'a jamais livré d'interprétation systématique de la notion de «nature» des actes individuels, son guide principal dans l'affirmation ou non de l'existence d'une obligation de motiver ayant toujours été la volonté de soumettre l'administration au contrôle des motifs. Par conséquent, sa démarche en matière de motivation a pu être décrite par un de ses vice-présidents dans les termes suivants:
Le vrai critère des actes administratifs ayant à être motivés ne peut pas être donné a priori, car, malgré les énonciations jurisprudentielles, il ne réside pas dans la «nature» de l'acte mais il émane a posteriori du doute que le juge peut avoir sur la légalité d'un acte dont l'auteur dissimule les motifs, en combinaison avec le caractère suffisant ou insuffisant des éléments versés au dossier. Si ces éléments sont susceptibles d'expliquer la mesure prise par l'administration, le juge a tendance à estimer que l'acte considéré n'a pas à être motivé. En l'absence d'éléments pouvant expliquer la mesure administrative, le juge annule l'acte en cause pour défaut de motifs en jugeant que cet acte nécessite, de par sa nature, d'être motivé ${ }^{25}$.

Certes, l'effort du Conseil d'État grec d'assurer ainsi le respect de la légalité administrative était fondamentalement animé par le souci de protéger les justiciables, les actes individuels concernés par l'exigence de motivation étant ceux qui menacent le plus les droits et libertés de ces derniers: les actes discrétionnaires par le risque d'arbitraire qu'ils contiennent par définition, les actes défavorables dans la mesure où ils posent des restrictions aux intérêts ou libertés des administrés et, enfin, les actes dérogatoires en ce qu'ils peuvent revêtir un aspect défavorable mais aussi parce qu'ils risquent de porter atteinte au principe d'égalité. M. Stassinopoulos, qui fut président du Conseil d'État grec, observait que, en vue d'affirmer le caractère obligatoire de la motivation administrative,

Il faut dans chaque cas prendre en considération le doute qu'un acte crée chez le juge quant à sa légalité ainsi que le préjudice que l'application de cet acte pourrait causer aux intérêts des particuliers ${ }^{26}$.

Cependant, la protection envisagée pour les administrés dans le cadre de la théorie jurisprudentielle en cause était exclusivement contentieuse; en atteste le fait que la motivation imposée par la nature de l'acte ne s'accomplit pas par l'indication formelle des motifs dans le corps de l'acte, mais elle peut ressortir des pièces du dossier. S'agissant en effet de la question de savoir comment l'organe administratif doit s'acquitter de son obligation de motiver ses actes nécessitant une motivation au titre de leur nature, le Conseil d'État grec a procédé à la distinction suivante: l'indication formelle des motifs dans le corps de l'acte n'est imposée que lorsque l'exigence de motivation résulte d'une prescription législative ${ }^{27}$. En revanche, concernant l'exigence jurisprudentielle de motivation en raison de la nature de l'acte, il suffit que les motifs se dégagent

22. Ces actes sont répertoriés, entre autres, par S. Délicostopoulos, $H \pi \alpha \rho \alpha \dot{\alpha} \beta \alpha \sigma \iota$ ov [La violation des formalités substantielles en tant que moyen d'annulation des actes administratifs], Athènes, Alpha Delta, 1970, p. 131 sq.;

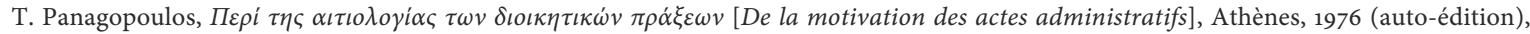
p. 85 sq.; et G. Ghérapetritis, La motivation des actes administratifs en Grèce..., p. 75 sq.

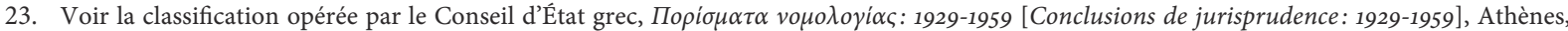
Imprimerie nationale, 1961, p. 183 sq.

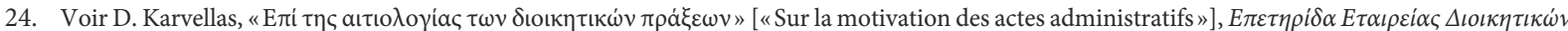

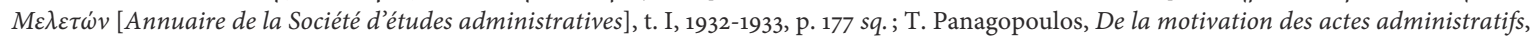
p. 84 sq.; S. Délicostopoulos, La violation des formalités substantielles..., p. 125; G. Ghérapetritis, La motivation des actes administratifs en Grèce..., p. 75 ; et A. Tsoutsos, «La motivation des actes administratifs», p. 127.

25. A. Tsoutsos, «La motivation des actes administratifs», p. 128.

26. M. Stassinopoulos, Traité des actes administratifs, Athènes, Institut français d'Athènes (Collection de l'Institut français d'Athènes), 1954, p. 205.

27. Voir par exemple CEgr, 59/1930, 131/1945, 397/1945 et 553/1950. 


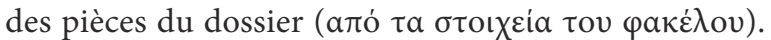
Le «dossier», une notion particulière du contentieux administratif grec, se réfère à l'ensemble des documents ou des autres éléments relatifs à une affaire litigieuse, $\mathrm{y}$ compris le rapport dans lequel l'administration explicite sa position sur l'affaire et répond aux moyens invoqués par le requérant. La loi prévoit à la charge de l'autorité administrative qui a édicté l'acte litigieux l'obligation de transmettre le dossier en cause au juge rapporteur dans les délais prévus ${ }^{28}$. Ce n'est donc que dans la phase contentieuse que le dossier de l'affaire apparaît comme un ensemble de pièces et d'éléments tenant lieu de motivation de l'acte contesté ${ }^{29}$. En dehors du contentieux, il n'existe pas pour les administrés de possibilité de consultation d'un tel dossier. Il leur est seulement loisible de consulter divers documents au titre du droit général d'accès aux documents administratifs ${ }^{30}$. Dès lors, l'idée de motivation ressortant du dossier illustre le fait que la préoccupation centrale dans la mise en œuvre de la théorie des actes nécessitant une motivation en raison de leur nature consistait à assurer l'efficacité du contrôle juridictionnel plutôt que la protection et l'information extra-juridictionnelles des administrés.

Eu égard à son mode d'accomplissement, la motivation des actes imposée par leur nature semble se rapprocher de la communication des motifs au juge à la française. Il est néanmoins à noter que, si avec l'exigence de motivation en cause le Conseil d'État grec entendait accroître son contrôle sur les motifs administratifs, ce n'était pas au titre d'un pouvoir inquisitorial particulier créé par la voie prétorienne. Le pouvoir général d'instruction du Conseil d'État grec, consacré dans les textes depuis le début de l'activité de ce dernier, lui permet de toute façon de prescrire à l'administration de lui apporter tout élément supplémentaire nécessaire de preuve, si les pièces du dossier s'avèrent insuffisantes pour la formation de la conviction du juge ${ }^{31}$.

La détermination in concreto du domaine d'application de l'exigence de motivation déduite de la nature d'un acte individuel, en fonction du besoin de protection juridictionnelle du justiciable, et, surtout, son mode d'accomplissement qui consiste non dans l'inscription des motifs dans le corps de l'acte mais dans leur inclusion dans les pièces du dossier contentieux, posent la question du contenu exact et, en fin de compte, de la nature même de cette exigence de motivation.

\section{B. La nature particulière de l'exigence de motivation des actes individuels}

Selon que l'obligation de motiver se fonde sur une prescription législative ou sur la nature de l'acte, le Conseil d'État grec distingue entre la motivation formelle indiquée dans le corps de l'acte et la motivation qui ressort des pièces du dossier. Or la source de l'obligation pour l'administration de motiver ses actes n'intéresse pas seulement le mode d'accomplissement de cette obligation, mais elle est à l'origine d'une distinction touchant à la nature même de l'exigence de motivation. La doctrine publiciste grecque admet généralement que, lorsqu'elle est imposée par une disposition législative, la motivation représente une formalité externe de l'acte et son accomplissement irrégulier constitue un vice de forme. En revanche, lorsque l'exigence de motiver est dégagée par le juge compte tenu de la nature de l'acte, la motivation requise relève de la légalité interne de l'acte administratif, son éventuelle irrégularité entraînant alors l'annulation de cet acte pour violation de la loi ${ }^{32}$.

Il s'ensuit que le palliatif de l'absence d'une obligation générale de motivation des actes individuels qu'a trouvé la jurisprudence grecque dans la théorie des actes nécessitant une motivation en raison de leur nature repose sur un glissement de la réflexion du registre de la forme vers celui du fond des actes individuels. Alors que le concept des actes nécessitant une motivation en raison de leur nature a été forgé pour viser les actes non soumis à une exigence de motivation ex lege, il a eu pour effet d'élargir non pas l'ouverture du vice de forme, mais celle de la violation de la loi. Le remède apporté par le Conseil d'État grec aux inconvénients résultant du principe de nonmotivation des actes individuels, à savoir l'obstruction du contrôle juridictionnel sur la légalité interne, n'a pas consisté dans l'instauration, par la voie prétorienne, d'une obligation occasionnelle d'indication formelle des motifs, mais dans l'exigence que le caractère justifié de l'acte déféré au contrôle du recours pour excès de pouvoir se déduise des éléments du dossier.

Dans ces conditions, l'exigence de motivation imposée par la nature d'un acte et dégagée par le juge est dépourvue de tout caractère formel. L'exigence en cause ne crée à la charge de l'administration aucune obligation relative à la confection de l'instrumentum de ses actes individuels. Elle

28. Voir l'article 23-2 du décret 18/1989 relatif au Conseil d'État grec (anciennement, article 23-2 du décret législatif 170/1973 et articles 49 et 55 de la loi codificatrice $3713 / 1928$ ).

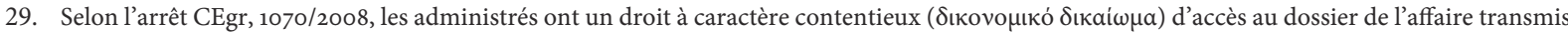
par l'administration au juge rapporteur à la suite de l'exercice d'un recours contentieux.

30. Réglementé actuellement à l'article 5 du Code grec de procédure non contentieuse et auparavant à l'article 16 de la loi $1599 / 1986$.

31. Le procès administratif est en Grèce de caractère inquisitorial, comme en France. À la différence du système français, les textes ont toujours expressément confié au Conseil d'État grec le pouvoir d'ordonner des mesures d'instruction dans le cadre du recours pour excès de pouvoir. Voir, anciennement, les articles 40-1, 49-7 et 8 et 55-1 de la loi codificatrice 3713/1928 et l'article 33 du décret législatif 170/1973 et, actuellement, l'article 33 du décret $18 / 1989$.

32. Voir, à titre indicatif, G. Ghérapetritis, La motivation des actes administratifs en Grèce..., p. 91; S. Délicostopoulos, La violation des formalités

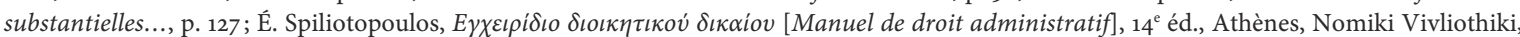

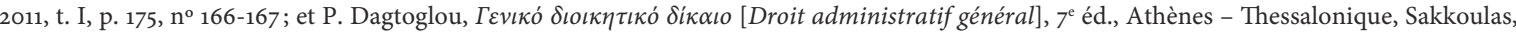
2015, p. 287-288, no $641-642$. 
n'établit pas non plus pour l'administration d'obligation contentieuse particulière concernant la préparation $\mathrm{du}$ dossier de l'affaire et sa communication au juge, étant donné que ces obligations ont toujours été spécialement prévues dans les dispositions du contentieux du recours pour excès de pouvoir. Le juge du recours pour excès de pouvoir est par ailleurs expressément doté par la loi d'un pouvoir général d'instruction en vertu duquel il peut réclamer à l'administration de fournir tout élément de preuve ou tout document supplémentaire pour compléter le dossier de l'affaire ${ }^{33}$.

En raison de son lien avec le caractère justifié de l'acte individuel litigieux, la motivation requise au titre de la nature des actes semble plutôt se rapprocher d'une exigence matérielle. Les pièces du dossier ne doivent pas simplement indiquer les motifs administratifs, mais ils doivent, de surcroît, en prouver la justesse. La motivation apparaît ainsi comme coïncidant avec la justification du

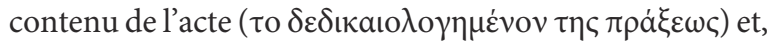

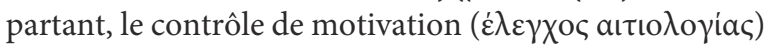
auquel se livre le juge administratif grec à partir des pièces du dossier ne porte pas sur l'accomplissement adéquat de l'exigence de motivation en tant qu'élément formel de l'acte considéré, en d'autres termes sur la légalité externe, mais sur la légalité interne et plus précisément sur les motifs de cet acte. C'est pourquoi l'annulation éventuelle

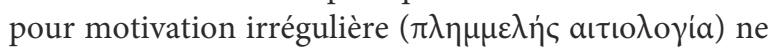
relève pas du vice de forme, mais de la violation de la loi.

Se fondant sur ces considérations, la doctrine grecque décrit la motivation imposée par la nature des actes comme un élément de la légalité interne des actes individuels et comprend l'exigence jurisprudentielle de motivation comme une exigence de justesse matérielle des motifs. Une telle approche laisse pour autant entière la question de savoir en quoi consiste exactement l'obligation qui pèse sur l'administration lorsqu'un acte est qualifié comme nécessitant une motivation en raison de sa nature. Comprise comme une exigence de justesse matérielle des motifs des actes individuels, l'obligation de motivation ne diffère pas de l'exigence générale, émanant du principe de légalité, que les décisions administratives soient prises sur la base des éléments de l'affaire et conformément à la règle de droit. Elle n'ajoute rien non plus aux règles matérielles qui régissent l'exercice du pouvoir discrétionnaire en droit grec, dont notamment les principes

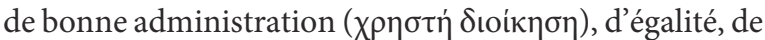
proportionnalité et de confiance légitime de l'administré

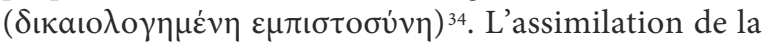
motivation imposée par la nature d'un acte à une exigence de justesse matérielle des motifs enlève à la construction jurisprudentielle sa particularité et ne justifie pas la conception des actes nécessitant une motivation en raison de leur nature comme consistant en une catégorie distincte d'actes administratifs.

Pour cerner l'exigence de motivation administrative en raison de la nature des actes, il est nécessaire de se rappeler le cadre dans lequel cette exigence a été élaborée. La raison pour le développement de la théorie jurisprudentielle en cause était l'extension du contrôle exercé sur les actes individuels discrétionnaires dans le cadre du recours pour excès de pouvoir. Le Conseil d'État grec a donc mis en place un moyen particulier de contrôle des motifs des actes discrétionnaires ${ }^{35}$. Dans cette optique, il n'a pas créé une règle destinée à produire des effets lors de l'exercice par l'administration de son pouvoir discrétionnaire, et qui relèverait donc du droit administratif matériel, mais il a redéfini l'étendue de son propre pouvoir de contrôle des actes discrétionnaires, ce qui touche au droit du contentieux du recours pour excès de pouvoir. Plus précisément, l'exigence de motivation dégagée de la nature des actes exprime essentiellement une technique de contrôle contentieux ${ }^{36}$. Le " contrôle de motivation " auquel se livre le juge grec du recours pour excès de pouvoir en cas d'actes nécessitant d'être motivés en raison de leur nature est un contrôle de légalité interne, susceptible de recouvrir, sans que la distinction ne soit faite expressément, la détection de divers vices de fond entachant l'acte litigieux, tels que l'erreur de droit, l'erreur de fait, la qualification juridique erronée ou le mauvais usage du pouvoir discrétionnaire ${ }^{37}$. En ce sens, le contrôle de motivation n'est pas un exemple de clarté méthodologique jurisprudentielle ${ }^{38}$, mais il sert pour autant de frein juridictionnel aux différentes facettes que peut revêtir l'illégalité administrative.

Si le Conseil d'État grec arrive ainsi à étendre son pouvoir de contrôle sur l'administration, il le fait dans des limites qu'il se pose lui-même, soucieux de ne pas vider de sa substance le pouvoir d'appréciation imparti par la loi à l'administration. En effet, en cas d'annulation d'un acte individuel pour motivation irrégulière $(\pi \lambda \eta \mu \mu \varepsilon \lambda \eta \dot{~} \varsigma$

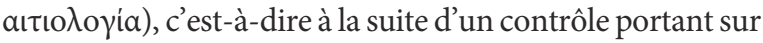

33. D. Petroulias, «Note sur la motivation des actes administratifs en droit grec», in Sur la forme et la procédure de l'acte administratif, G. Dupuis (dir.), Paris, Economica, 1979, p. 39, observe que, pour obliger l'administration à lui expliciter ses motifs, le juge pourrait tout simplement exciper du caractère inquisitorial de la procédure contentieuse au lieu d'invoquer comme fondement la théorie des actes nécessitant une motivation en raison de leur nature.

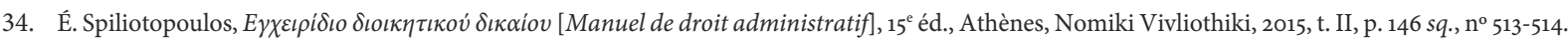

35. Voir les mots attentivement choisis de M. Stassinopoulos, Traité des actes administratifs, p. 126: "[Il y a lieu de] distinguer entre la mention des motifs prescrite par la loi et celle imposée par la nature de l'acte. Dans le premier cas il s'agit d'une forme, alors que dans le second cas il est seulement question de contrôle de légalité de l'acte du point de vue du fond».

36. Selon une autre approche, la théorie des actes nécessitant une motivation en raison de leur nature ne fait que renverser, au détriment de l'administration, la charge de la preuve lors du contrôle juridictionnel des actes individuels: I. Kambitsis, «To $\beta a ́ \rho \circ \varsigma$ a

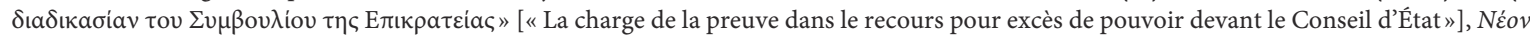
Síkaıv [Droit nouveau], 1963, p. 180 sq.

37. G. Ghérapetritis, La motivation des actes administratifs en Grèce..., p. 144-145.

38. D. Petroulias, «Note sur la motivation...», p. 40. 
la légalité interne, il est loisible à l'administration d'édicter un nouvel acte ayant le même contenu, mais en le complétant cette fois-ci de la motivation appropriée, à savoir d'un dossier plus convaincant ${ }^{39}$. De l'annulation pour motivation irrégulière, prononcée après un contrôle portant sur la justesse des motifs de l'acte, sont dès lors essentiellement tirées les conséquences de l'annulation pour vice de forme ${ }^{40}$. Les effets limités qui résultent pour l'administration des arrêts prononçant l'annulation pour motivation irrégulière peuvent être perçus comme un indice de l'existence d'une certaine autonomie de ce moyen d'annulation par rapport au moyen d'annulation pour violation de la loi, même si le premier est habituellement présenté comme une souscatégorie du second ${ }^{41}$. En annulant un acte pour motivation irrégulière, le juge accorde à l'administration une nouvelle occasion de réexaminer une affaire dont le traitement initial ne l'a pas totalement convaincu.

En revanche, l'annulation pour violation de la loi prive l'administration de toute possibilité de revenir sur l'affaire par l'édiction d'un acte ayant le même contenu que l'acte initial. Sur ce point, il est intéressant de noter que le Conseil d'État grec recourt à l'annulation pour violation de la loi quand, après des annulations successives pour motivation irrégulière et des renvois subséquents à l'administration dans le cadre d'une même affaire, l'administration réitère en édictant un acte au même contenu sans aucune amélioration du dossier l'accompagnant. Le juge en déduit alors que l'administration agit pour des motifs obscurs et très probablement illégaux et met fin au va-et-vient entre l'administration et lui-même en prononçant l'annulation pour mauvais usage du pouvoir discrétionnaire (какท́

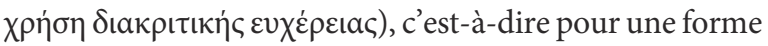
de violation de la loi, ce qui empêche définitivement l'administration de réémettre un acte avec le même contenu ${ }^{42}$.

En conclusion, le contrôle de motivation et, partant, le moyen d'annulation relatif à la motivation irrégulière constitue un outil flexible à l'aide duquel le juge grec régule l'étendue de son contrôle sur le terrain du pouvoir administratif discrétionnaire, selon des considérations souvent plus pragmatiques que juridiques. Dans tous les cas, par la théorie des actes nécessitant une motivation en raison de leur nature, le Conseil d'État grec n'a jamais entendu créer à la charge de l'administration une obligation particulière d'extérioriser ses motifs, et encore moins de créer au bénéfice des administrés un droit spécial, contentieux ou non contentieux, de prendre connaissance des motifs administratifs. Cependant, cette jurisprudence a contribué, plus généralement, à établir en droit grec une conception assez confuse de la motivation administrative.
Il en est ainsi car, par sa théorie des actes nécessitant une motivation en raison de leur nature, le Conseil d'État grec a créé un nœud insoluble ${ }^{43}$ entre la motivation en tant qu'exigence de légalité externe et les motifs de l'acte administratif. Conçue par contraste avec l'obligation ex lege de motivation dans l'arrêt de principe 59/1930, l'exigence de motivation en raison de la nature d'un acte aurait dû logiquement instaurer pour les actes qu'elle concernait une nouvelle cause de vice de forme. Mais par l'exigence en question le juge grec établissait en réalité pour les actes concernés un nouveau moyen d'annulation, proche de celui de la violation de la loi. La référence à la motivation formelle requise par la loi dans cet arrêt exprimait tout simplement la préoccupation du juge que l'absence d'une obligation législative pour l'administration d'indiquer ses motifs ne conduise pas à ce que l'acte échappe à tout contrôle juridictionnel quant à sa légalité interne.

L'arrêt d'Assemblée 78/1930, rendu par le Conseil d'État grec très peu de temps après l'arrêt de principe 59/1930, contient à propos de l'exigence jurisprudentielle de motivation au titre de la nature de l'acte un considérant dont la formulation est très caractéristique. Faisant valoir que

L'absence de motivation peut entraîner l'annulation d'un acte administratif soit lorsque la loi impose expressément la motivation soit lorsque, s'agissant d'un acte administratif nécessitant une motivation en raison de sa nature, le caractère justifié de l'acte ne se dégage ni de l'activité administrative dans son ensemble ni des documents fournis; l'absence de motivation entrave alors le contrôle portant sur l'exercice légal, par l'organe administratif, de son pouvoir discrétionnaire $[\ldots]$

le Conseil juxtaposait immédiatement l'exigence jurisprudentielle de motivation dégagée de la nature d'un acte à la motivation imposée ex lege.

Les arrêts 59/1930 et 78/1930 aussi bien que leurs suites ont été appréhendés en droit grec comme mettant en relation binaire la motivation ex lege et l'exigence jurisprudentielle de motivation ressortant du dossier. Les actes ne nécessitant pas de motivation en vertu d'une disposition de loi étaient «en revanche» susceptibles de tomber sous le coup de l'exigence jurisprudentielle de motivation à partir du dossier. Destinée à s'appliquer aux actes dont la loi n'avait pas prévu la motivation obligatoire, la théorie des actes nécessitant une motivation en raison de leur nature a dès lors pu être regardée comme une exception au principe de non-motivation des actes individuels ${ }^{44}$, même si elle ne posait aucune exigence d'extériorisation des motifs, et encore moins une exigence de motivation

39. D'une jurisprudence abondante, voir CEgr, 2453/1984, 4370/1988, Ass., 2611/1989, 2675/1996, 2295/2002, 1104/2004, 494/2006, 481/2008, 50/2009, 105/2010 et $158 / 2010$.

40. P. Vegléris, "Caractéristiques du Conseil d’État... », p. 35, évoque une absorption de la motivation imposée par la nature d’un acte dans le régime de la motivation formelle.

41. Voir par exemple É. Spiliotopoulos, Manuel..., t. II, p. 150, $\mathrm{n}^{\circ} 516$.

42. Voir par exemple CEgr, Ass., 1820/1989, 5417/1995, 710/2001, 2705/2004, 379/2007, 220/2009 et 858/2011.

43. P. Vegléris, «Caractéristiques du Conseil d'État...», p. 32.

44. En ce sens, voir A. Tsoutsos, «La motivation des actes administratifs», p. 127; G. Ghérapetritis, La motivation des actes administratifs en Grèce..., p. 75 sq. ; et É. Spiliotopoulos, Manuel..., t. I, p. 175, nº 166. Contra, voir D. Petroulias, "Note sur la motivation...», p. 36 sq. 
formelle, et que le contrôle « de motivation » auquel elle conduisait portait exclusivement sur la légalité interne de l'acte concerné.

Cette jonction de la motivation imposée par la loi et de la motivation déduite par la nature des actes a eu des incidences importantes sur la compréhension générale de la motivation administrative en Grèce. Une conception trop large de la «motivation» ( $\alpha \iota \tau \iota$ olo jí $\alpha)$ s'est établie en droit grec, de sorte que ce terme comprend non seulement l'exigence formelle d'indication des motifs, mais aussi les motifs des actes administratifs et leur justesse ${ }^{45}$. De façon symétrique, le terme "contrôle de motivation" ( $\dot{\varepsilon} \lambda \varepsilon \gamma \chi \bigcirc \circ$

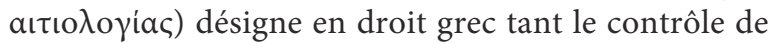
légalité externe portant sur le respect de la motivation en tant qu'élément formel de l'acte individuel que le contrôle des motifs suscité par la qualification d'un acte comme nécessitant une motivation en raison de sa nature.

En outre, la doctrine grecque considère que l'obligation de motivation peut avoir alternativement comme fondement une disposition expresse de la loi ou bien la jurisprudence, lorsqu'elle est imposée par la nature de l'acte $^{46}$. La perception de la motivation formelle et de l'exigence jurisprudentielle de motivation ressortant des pièces du dossier comme étant deux aspects du même phénomène est illustré par le fait que le régime et les modalités d'accomplissement des deux exigences de motivation sont, eux aussi, généralement traités de manière indifférenciée. La motivation est décrite, dans tous les cas, comme devant être spécifique, claire et adéquate et comme devant se référer exclusivement à des motifs préexistants à l'édiction de l'acte ${ }^{47}$. La seule distinction résiderait dans le fait que la motivation requise par prévision de la loi doit être inscrite dans le corps de l'acte, alors que la motivation imposée par la nature de l'acte peut ressortir des pièces du dossier. L'adhérence de la doctrine publiciste grecque à ce binôme, constitué de la motivation en tant qu'exigence formelle et de la motivation au sens de la justification matérielle de l'acte, est d'autant moins compréhensible que la même doctrine admet volontiers la différence de nature entre les deux types de motivation ${ }^{48}$ et reconnaît, d'un point de vue systématique, que la motivation relève tantôt du droit de la procédure administrative non contentieuse et tantôt du droit administratif matériel ${ }^{49}$.

Par la théorie des actes nécessitant une motivation en raison de leur nature, le Conseil d'État grec a fini par enchevêtrer la question de la motivation comme un élément de la légalité externe des actes administratifs et la question du contrôle des motifs. Le binôme dans lequel ont été imbriquées ces deux questions a fonctionné au détriment de la motivation formelle. Dans la mesure où la théorie susmentionnée assure aux administrés une protection juridictionnelle satisfaisante de leurs droits et libertés, l'intérêt des administrés à la connaissance non contentieuse des motifs administratifs est considéré comme moindre. La place de la motivation formelle en tant que garantie de la procédure administrative non contentieuse est alors restée secondaire en droit grec.

\section{La place secondaire réservée à la motivation formelle des actes individuels}

Le renforcement de la protection juridictionnelle des droits et libertés par le biais de la théorie jurisprudentielle des actes nécessitant une motivation en raison de leur nature a conduit le droit grec à se désintéresser de la question de la connaissance des motifs des actes individuels en tant que droit de procédure administrative non contentieuse complémentaire de la garantie contentieuse (A). Dans ce cadre, l'apport du Code grec de procédure administrative non contentieuse à l'affirmation du droit à la motivation formelle a été lui aussi très restreint (B).

\section{A. Le désintérêt pour l'aspect non contentieux de la motivation}

À propos des actes non soumis à une obligation de motivation en vertu de la loi, la jurisprudence administrative grecque n'a dégagé presque aucune exigence d'extériorisation formelle des motifs. Aussi l'obligation de motivation en tant qu'élément formel des actes individuels est-elle toujours demeurée exceptionnelle en droit grec et tributaire de l'existence d'une prévision législative. Bien plus, à la différence de ce qui s'est passé en France à la veille de la loi du 11 juillet 1979, l'idée du renforcement de l'exigence de motivation formelle des actes individuels a rarement été avancée dans le débat juridique grec.

Pourtant, la signification de la motivation pour les administrés, indépendamment de leur qualité éventuelle de justiciables, et son rôle protecteur au stade procédural

45. Pourtant, dans un de ses premiers arrêts (204/1929) le CEgr se référait aux «faits justificatifs» de l'acte, aux «causes» de l'action administrative, et aux «mobiles relatifs à l'intérêt du service» pour désigner ce qui ensuite a été couvert par le terme unique de "motivation». Ces termes n'ont pas trouvé racine dans la jurisprudence et c'est le terme «motivation» qui a prévalu (P. Vegléris, "Caractéristiques du Conseil d'État...", p. 29).

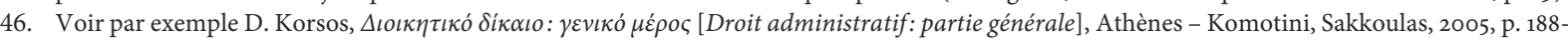

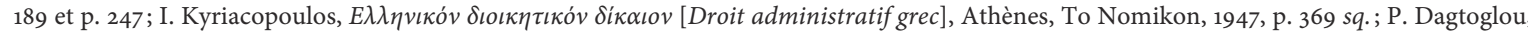
Droit administratif général, p. 287, nº 640; É. Spiliotopoulos, Manuel..., t. I, p. 175, n 166-167; T. Panagopoulos, De la motivation..., p. 25 sq.; S. Délicostopoulos, La violation des formalités substantielles..., p. 120 sq.; A. Tsoutsos, "La motivation des actes administratifs», p. 127 sq.; et G. Ghérapetritis, La motivation des actes administratifs en Grèce..., p. 73 sq.

47. Voir tous les auteurs de la note précédente.

48. Voir M. Stassinopoulos, Traité des actes administratifs, p. 126; A. Tsoutsos, «La motivation des actes administratifs», p. 127; S. Délicostopoulos, La violation des formalités substantielles..., p. 120 sq.; D. Petroulias, "Note sur la motivation... », p. 32 sq.; G. Ghérapetritis, La motivation des actes administratifs en Grèce..., p. 73 sq.; É. Spiliotopoulos, Manuel..., t. I, p. 175, n 166-167; et D. Korsos, Droit administratif..., p. 189 sq.

49. Voir T. Panagopoulos, De la motivation..., p. 29-30; S. Délicostopoulos, La violation des formalités substantielles..., p. 119 sq. 
ou précontentieux ne sont pas inconnus du Conseil d'État grec. Déjà dans son arrêt 923/1931, ce dernier estimait que, outre la facilitation du contrôle juridictionnel, l'exigence de motivation s'impose également pour assurer que

Le pouvoir discrétionnaire s'exerce conformément à la véritable volonté du législateur, qui a instauré ce pouvoir non comme un acte arbitraire mais comme une décision juste, prise dans l'intérêt réel de l'administration.

Cependant, le Conseil d'État grec a manifesté la volonté de rendre la décision administrative plus lisible pour les administrés et d'améliorer l'information de ces derniers sur les motifs administratifs uniquement dans les domaines de la sécurité sociale et de la sélection du personnel de la haute fonction publique, dans lesquels il a dégagé l'obligation pour l'administration de motiver expressément certains de ses actes. Ainsi, il a exceptionnellement admis que, s'agissant des décisions édictées par les organismes de sécurité sociale relatives aux prestations légales à leurs assurés ou aux ayants droit, les motifs doivent figurer sur le corps de l'acte, le renvoi aux pièces du dossier n'étant pas suffisant $^{50}$. Plus récemment, il a imposé à l'administration l'obligation de rédiger, en tant que formalité substantielle de la procédure de promotion des fonctionnaires au poste de chef de direction générale, le procès-verbal des entretiens oraux avec les candidats, qu'elle doit incorporer dans sa décision finale ou joindre à cette dernière, en vue de garantir l'impartialité et l'exercice légal, par l'administration, de son pouvoir discrétionnaire ${ }^{51}$.

Il est à noter que l'intérêt principalement contentieux dont le droit grec investit l'extériorisation des motifs administratifs est également visible au niveau du raisonnement constitutionnel. Dans la jurisprudence du Conseil d'État grec, l'exigence de motivation administrative, prise au sens large que lui confère le droit grec, est systématiquement fondée sur le droit à la protection juridictionnelle, consacré à l'article 20-1 de la Constitution de 1975, ainsi que sur la compétence générale du Conseil d'État grec pour connaître des recours pour excès de pouvoir exercés à l'encontre des actes administratifs, sur la base des articles 94-1 et 95-1 alinéa $\alpha$ ) de la Constitution. C'est de manière complémentaire que la motivation est associée, le cas échéant, aux principes d'État de droit, de liberté, d'égalité d'accès aux emplois publics et de transparence administrative, consacrés respectivement aux articles 25-1, 5-1, 4-4 et 103-7 de la Constitution. Dès lors, la motivation administrative est reconnue comme étant une exigence constitutionnelle surtout parce qu'elle conditionne l'efficacité du contrôle contentieux et, secondairement, en tant que garantie en faveur des administrés pendant le processus d'élaboration des actes individuels ${ }^{52}$.

De son côté, la doctrine grecque n’a jamais hésité à reconnaître le rôle protecteur joué par la motivation obligatoire dès la phase de l'élaboration des actes individuels, en dehors de son incontestable fonction diagnostique, c'est-à-dire sa contribution à la détection des irrégularités matérielles de ces actes par le juge ou par les administrés eux-mêmes en vue de l'exercice d'un recours ${ }^{53}$. Les auteurs grecs partagent généralement les réflexions françaises ayant trait à l'apport de l'obligation d'indication formelle des motifs au respect de la légalité, à la diminution du risque d'arbitraire et, partant, à la protection préventive des droits et intérêts des personnes concernées par l'acte projeté. L'utilité de la connaissance des motifs d'un acte individuel pour l'exercice plus efficace du droit à la protection juridictionnelle paraît également fondamentale.

Or, avant l'adoption du Code grec de procédure administrative non contentieuse en 1999, et malgré cette reconnaissance de l'utilité non contentieuse de la motivation, la doctrine grecque ne tenait pas particulièrement à réclamer le renforcement de l'obligation non contentieuse pour l'administration d'extérioriser ses motifs, surtout parce que la garantie juridictionnelle des droits des administrés à travers le contrôle des motifs lui paraissait suffisante ${ }^{54}$. De ce point de vue, et s'agissant de la protection des administrés, le droit grec préférait s'en remettre au juge encore davantage que le droit français, dont la tendance à sous-estimer les garanties préalables au bénéfice de la protection contentieuse a été pendant longtemps déplorée. La confiance du droit grec dans la voie juridictionnelle de la protection des droits et libertés au détriment de la voie préalable et non contentieuse n'était d'ailleurs pas sans relation avec la crainte d'une éventuelle multiplication des annulations pour vice de forme en cas d'extension du droit des administrés à la connaissance non contentieuse des motifs ${ }^{55}$, crainte qui avait également été exprimée dans la doctrine française avant la loi du 11 juillet 1979. En outre, la possibilité pour les administrés d'accéder aux documents administratifs, selon d'abord un principe général du droit dégagé par le juge administratif grec et ensuite en vertu de l'article 16 de la loi 1599/1986, pouvait pallier le problème de la mauvaise information des administrés résultant de l'obligation seulement exceptionnelle de motivation formelle des actes individuels.

Une certaine réticence envers le renforcement du droit des administrés à la connaissance non contentieuse des motifs administratifs caractérisait les principales tentatives de codification du droit de la procédure non contentieuse qu'a connues la Grèce avant l'introduction

50. CEgr, 804/1971 et 42/1971.

51. CEgr, 690-693/2017, 865/2015, 2162/2014, 3766/2012, 148-149/2011, Ass., 3052 et 3058/2009.

52. Pour davantage d'informations, voir V. Kapsali, Les droits des administrés dans la procédure administrative non contentieuse. Étude comparée des droits français et grec, Issy-les-Moulineaux, LGDJ (Bibliothèque de droit public; 291), 2015, p. 164 sq., $\mathrm{n}^{\circ} 177$ sq.

53. Voir par exemple G. Ghérapetritis, La motivation des actes administratifs en Grèce..., p. 25 sq.; T. Panagopoulos, De la motivation..., p. 68 sq. et p. 102 sq.; et D. Karvellas, «Sur la motivation...», p. 176.

54. D. Petroulias, «Note sur la motivation... », p. 31-32; S. Délicostopoulos, La violation des formalités substantielles..., p. 149.

55. En ce sens, voir S. Délicostopoulos, La violation des formalités substantielles..., p. 126-127. 
du Code de 1999. Dans l'avant-projet de Code élaboré à titre personnel par M. Stassinopoulos en $1967^{56}$, l'article 62 prévoyait que tous les actes individuels qui portent un préjudice à des intérêts individuels, et notamment les actes de retrait et ceux qui s'opposent à des avis ou des rapports, doivent comporter une motivation. Toutefois, s'agissant du mode d'accomplissement de l'obligation de motivation, il était prévu que la motivation pouvait ressortir des documents sur lesquels s'appuie ou auxquels se réfère l'acte administratif, à l'exception des cas où l'exigence de motivation découle d'une disposition spéciale de la loi ; les motifs devraient alors être indiqués sur le support même de l'acte. La prévision spéciale dans la loi était dès lors maintenue en tant que condition pour le caractère formel de la motivation.

Le projet de Code de la commission constituée sous la présidence du professeur S. Flogaïtis, qui a été déposé en 1996 au Parlement en tant que projet de loi avant d'être retiré par la suite ${ }^{57}$, semblait tenir davantage compte des dimensions non contentieuse et formelle de la motivation. Selon l'exposé des motifs du projet de loi en cause, l'obligation de motivation des actes individuels résulte $\mathrm{du}$ principe d'État de droit et s'impose non seulement dans l'objectif de faciliter l'exercice du contrôle juridictionnel, mais également en vue de la meilleure information des particuliers dont les droits et libertés se trouvent restreints par les actes individuels, aussi bien que de la rationalisation de l'administration durant l'exercice de son pouvoir de décision. Dans son article 20-1 et 2, le projet préconisait alors la motivation obligatoire de tous les actes individuels écrits, et, en particulier pour les actes discrétionnaires, l'explicitation des critères employés pour leur édiction. Selon l'article 20-3, la motivation pouvait ressortir des pièces du dossier, mais avec l'inscription obligatoire, en parallèle, de ses éléments essentiels dans le corps même de l'acte individuel. En conséquence, ce projet de Code donnait un caractère formel général à la motivation requise. Mais il évitait en même temps de qualifier l'exigence de motivation de formalité externe des actes individuels. Sans doute en contradiction avec son propre article 20-1, ce projet contenait, dans l'article 20-5, une référence à des actes dont la motivation n'est imposée ni par la loi ni par leur nature. Il adoptait ainsi implicitement la distinction traditionnelle du droit grec entre la motivation comme un élément de la légalité externe des actes individuels, en cas de sa prévision spéciale dans la loi, et la motivation en tant qu'un élément de légalité interne, lorsqu'elle est imposée par la nature d'un acte.

Un esprit encore moins favorable à l'aspect non contentieux de la motivation marque le Code de procédure administrative non contentieuse qui a été finalement adopté en 1999 et dont l'apport à la consécration de la motivation formelle des actes individuels est très limité.

\section{B. L'apport limité de l'article 17 du Code de procédure administrative non contentieuse}

L'article 17-1 du Code grec semble renverser le principe de non-motivation des actes individuels. Toutefois, la généralisation de la règle de motivation administrative énoncée dans cette disposition n'est pas synonyme de généralisation de l'exigence d'une motivation formelle:

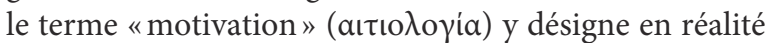
tant la motivation comme élément de la légalité externe de l'acte individuel qu'une exigence liée à la légalité interne. Autrement dit, en réglementant la question de la «motivation", l'article 17 se réfère à une exigence que le droit grec appréhende traditionnellement comme ayant une double nature.

De cette appréhension de la motivation témoigne d'abord le choix des auteurs du Code de traiter, dans l'article 17 , aussi de la question de la preuve dans la procédure administrative décisionnelle. Selon l'article 17-3,

Lorsque l'acte administratif est émis d'office, les éléments de preuve sont réunis à l'initiative de l'autorité compétente pour l'édiction de l'acte. Lorsque l'édiction de l'acte est demandée par l'intéressé, ce dernier doit fournir les pièces justificatives déterminées par la loi, sauf si ces pièces sont déjà en la possession de l'autorité compétente pour l'édiction de l'acte.

L'association de cette question à la garantie de motivation témoigne de la proximité de cette dernière, au sens du Code, avec la légalité interne de l'acte administratif. La réunion des éléments de preuve et la production des pièces justificatives ne concernent pas la confection de l'instrumentum d'un acte, mais les conditions légales d'édiction de l'acte. Le traitement de la question de la preuve dans une disposition relative à la motivation ne peut s'expliquer que si la motivation est comprise comme tenant également à la justification matérielle de l'acte considéré.

Surtout, l'appréhension large de la notion de motivation retenue dans le Code est illustrée par la façon dont les auteurs de ce dernier ont abordé la question du mode d'accomplissement. Si l'exigence de motivation concerne désormais tous les actes individuels, il n'est pas toujours indispensable que les motifs soient indiqués sur le support de l'acte. L'article 17-2 prévoit en effet que la motivation peut « ressortir des pièces du dossier, à moins que son inclusion dans le support formel de l'acte ne soit prescrite expressément par la loi». Ce choix du législateur n'est pas sans rappeler l'état du droit antérieur et le binôme établi, à partir de la jurisprudence relative aux actes nécessitant une motivation en raison de leur nature, entre la motivation ex lege, devant être inscrite dans le corps de l'acte, et la

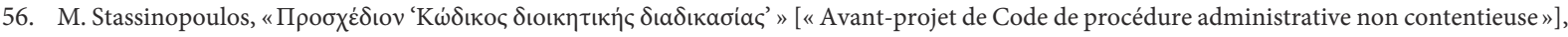

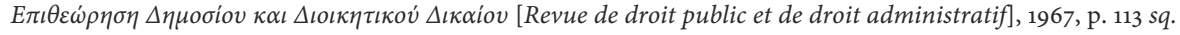

57. Pour le texte du projet de loi et son exposé des motifs, voir V. Karacostas, S. Pavlaki, Commentaire article par article..., p. 845 sq. 
motivation imposée par la nature de l'acte, pouvant être déduite des pièces du dossier. En conséquence, le renvoi de l'article 17-2 aux pièces du dossier n'est pas une simple règle de détermination du mode d'accomplissement de l'exigence de motivation, mais il se réfère à la nature même de cette exigence. Ce choix incite à penser que le Code n'a pas seulement reproduit l'ancienne règle jurisprudentielle, selon laquelle la motivation doit être incluse dans le support de l'acte individuel lorsqu'elle est imposée par une prévision spéciale de la loi, sinon elle peut ressortir des pièces du dossier, mais qu'il a incorporé la conception traditionnelle de la motivation en tant qu'une exigence à double nature. Dans cette optique, la motivation sur dossier n'est pas, au sens du Code, un type de motivation formelle dont le régime serait tout simplement moins exigeant par rapport à la motivation incluse dans le corps de l'acte, mais elle constitue une exigence de nature différente: sa méconnaissance conduit à l'annulation de l'acte non pour vice de forme, mais pour violation de la loi.

La lecture de l'article 17-2 par la doctrine dominante grecque va dans ce sens. Les auteurs sont unanimes pour admettre que, sous le régime du Code de procédure administrative non contentieuse, le terme "motivation" continue à désigner deux garanties: d'une part la formalité externe de l'acte individuel, lorsqu'elle doit être indiquée dans le corps de l'acte, et d'autre part une exigence relative à la légalité interne des actes individuels et dont les irrégularités entraînent l'annulation pour violation de la loi, lorsqu'elle peut simplement ressortir des pièces du dossier ${ }^{5}$. Le Conseil d'État grec confirme cette lecture de la disposition en cause ${ }^{59}$. Toutefois, la double nature assignée à l'exigence de motivation crée une importante incertitude quant aux conséquences normatives à tirer de la généralisation de celle-ci en vertu de l'article 17-1 du Code. La généralisation de la règle de motivation des actes individuels n'équivalant pas à une généralisation de la motivation formelle, la question se pose de savoir quel est le contenu normatif exact de la disposition en cause.

Une réponse envisageable consisterait dans l'idée selon laquelle l'article 17 fournit la consécration légale du binôme, issu de la jurisprudence du Conseil d'État grec, des actes nécessitant une motivation par prévision de la loi et des actes nécessitant une motivation en raison de leur nature. Cependant, cette approche se heurte au libellé même de l'article 17-1, qui, par l'emploi du singulier dans la phrase «l'acte administratif individuel doit comporter une motivation ", rend obligatoire la motivation de tous les actes individuels. Parmi les actes individuels, ceux dont la motivation est également prescrite par une disposition législative spéciale constituent simplement un sous-ensemble. C'est pourquoi une partie de la doctrine déduit de la formulation générale de l'article 17-1 que la distinction entre les actes nécessitant une motivation en raison de leur nature et les actes dont la nature n'impose pas la motivation est devenue sans objet ${ }^{60}$. Compte tenu de la formulation générale de l'article 17-1, on pourrait dès lors soutenir que le Code a introduit dans le droit administratif grec une nouvelle distinction fondamentale, entre les actes individuels dont la motivation est prescrite par une disposition spéciale de loi et le reste des actes individuels, dont la motivation est obligatoire en vertu de la disposition générale de l'article 17-1 du Code. Dans cette optique, et étant donné que le Code fournit désormais un fondement général à cet effet, il n'y aurait plus lieu pour le juge administratif de dégager lui-même l'exigence de motivation des actes individuels.

Néanmoins, cette vision des choses suscite des interrogations. Si le législateur grec a effectivement entendu établir une obligation générale de motivation des actes individuels, quel est le contenu exact de l'obligation qu'il a ainsi instaurée à la charge de l'administration? L'exigence de motivation qui s'accomplit par référence aux pièces du dossier ne concerne en rien, au sens de l'article 17-2 du Code, la confection de l'instrumentum des actes individuels, mais elle s'identifie quant à son contenu à l'exigence de motivation que la jurisprudence dégageait traditionnellement de la nature des actes. Dans ces conditions, sa proclamation en règle générale est dépourvue de sens. Si l'on appréhende l'exigence de motivation qui ressort des pièces du dossier comme une exigence de justesse matérielle des motifs, on est alors obligé de constater que l'article 17-1 du Code n'établit à la charge de l'administration aucune obligation spéciale ou différente ni par rapport à son obligation générale de respecter le principe de légalité ni par rapport aux principes qui régissent en particulier l'exercice de son pouvoir discrétionnaire. Si, d'autre part, on tente de comprendre l'exigence de motivation qui ressort des pièces du dossier au sens de l'article 17-2 du Code comme une règle de soumission des actes concernés au contrôle juridictionnel des motifs, la disposition de l'article 17-1 du Code ne fait que fournir le fondement légal du pouvoir du juge du recours pour excès de pouvoir d'exercer un "contrôle de motivation" de tous les actes individuels. Or une telle disposition paraît assez paradoxale quand elle figure dans un Code de procédure administrative non contentieuse.

Face à l'incertitude entourant le contenu normatif de l'article 17-1 du Code, le Conseil d'État grec préfère souvent recourir à sa propre jurisprudence relative aux actes nécessitant une motivation en raison de leur nature afin de se prononcer sur le caractère obligatoire de la motivation. Alors que l'article 17-2 du Code fait l'objet

58. Les manuels de droit administratif grec évoquent cette distinction selon des termes identiques à ceux qu'ils employaient avant 1999. Voir par exemple É. Spiliotopoulos, Manuel..., t. I, p. 175, n 166 ; et D. Korsos, Droit administratif..., p. 189 sq.

59. Voir par exemple CEgr, 2096/2002, 3897/2004, 690-693/2017 et 1173/2017.

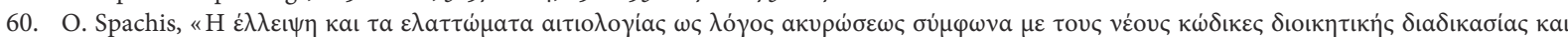

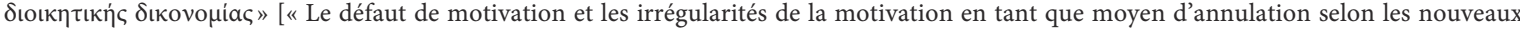

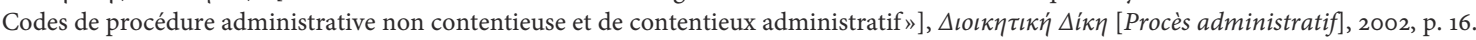


d'une invocation fréquente s'agissant pour le Conseil d'exclure l'existence d'une obligation formelle de motivation en l'absence de disposition spéciale de $l{ }^{6}{ }^{61}$, l'appel à l'article 17-1 s'agissant de fonder l'exigence de motivation est en revanche assez limité. Pour affirmer l'existence d'une exigence de motivation, le Conseil d'État grec n'hésite pas, le cas échéant, à invoquer la nature des actes déférés à son contrôle ${ }^{62}$. À l'inverse, il lui arrive aussi d'exciper de la nature de l'acte contesté afin d'exclure le caractère obligatoire de la motivation ${ }^{63}$. La théorie des actes nécessitant une motivation en raison de leur nature se trouve dès lors toujours applicable sous le régime du Code de procédure administrative non contentieuse ${ }^{64}$.

Il s'ensuit que le Code grec de procédure administrative non contentieuse a permis la survivance du binôme constitué de la motivation formelle, lorsqu'elle est prévue spécialement dans la loi, et de la motivation en tant qu'élément lié à la légalité interne de l'acte dans tous les autres cas. Il a à la fois permis la perpétuation de la conception essentiellement contentieuse de la motivation administrative et, avec elle, la dévalorisation de la motivation en tant qu'exigence formelle et en tant que garantie non contentieuse des droits et intérêts individuels. En effet, ainsi qu'il ressort de l'article 17-2 du Code, l'exigence de motivation formelle des actes individuels reste tributaire de sa prévision par des dispositions spéciales et, par conséquent, elle revêt toujours un caractère exceptionnel. Cela revient à dire que, sous le régime du Code, le principe de non-motivation des actes individuels est dans les faits toujours valable.

S'il n'a pas généralisé ni étendu le champ d'application de la motivation formelle des actes individuels, le Code grec de procédure administrative non contentieuse n'a pas non plus livré de réglementation générale du domaine de l'exigence en cause. Il est toujours nécessaire de rechercher les fondements de l'obligation de motivation formelle dans des dispositions sporadiques, extérieures au Code, ce qui est peu favorable à la sécurité et à la clarté juridiques auxquelles on peut légitimement s'attendre d'un tel Code ${ }^{65}$. En définitive, le champ d'application de la motivation formelle demeure, sous le régime du Code grec, non seulement limité mais aussi non clarifié. La réglementation d'ensemble, dans le sens d'une extension, du domaine de la motivation formelle reste donc une question ouverte. L'axe et la mesure de cette extension ne sauraient être autre chose que le besoin d'assurer la protection des droits et intérêts individuels face au pouvoir administratif unilatéral par des mécanismes également non contentieux.

61. Voir par exemple CEgr, 2096/2002, 4027/2004, 1955/2008, 3407/2008, 3830/2009, 208/2012 et 1390/2014.

62. Par exemple, il a pu admettre que nécessitent une motivation en raison de leur nature les actes relatifs à l'exercice des droits fondamentaux, comme la décision de soumission d'un immeuble à un régime restrictif en vue de la protection de l'héritage culturel (CEgr, 1180/2009), le refus de reconnaissance de l'équivalence d'un diplôme universitaire obtenu à l'étranger (CEgr, 2602/2010), la décision proclamant la déchéance de la nationalité grecque (CEgr, 2798-2800/2009) ou le rejet de la demande d'autoriser le fonctionnement d'une maison de culte (CEgr, Ass., 4022/2012), ainsi que divers actes ayant un caractère de sanction ou retirant des actes favorables (CEgr, 3457/2007, 2631/2009, 1350/2013).

63. Tel est par exemple le cas des actes émis à la suite de l'exercice d'une compétence liée (CEgr, 1120/2005, 2414/2009), de la notation des copies dans le cadre d'un concours administratif (CEgr, 3064/2011) ou des arrêtés du conseil des ministres relatifs à la promotion aux postes de président et

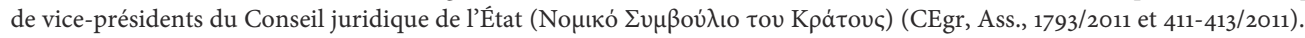

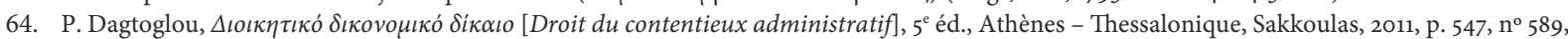
soulève l'appropriation problématique de l'article 17 du Code par la jurisprudence administrative.

65. En ce sens, voir T. Panagopoulos, De la motivation..., p. 65. 